\title{
Noncardiogenic pulmonary edema from massive irrigation fluid absorption during hysteroscopic myomectomy: a case report
}

\begin{abstract}
Background: Gynecological hysteroscopy is a relatively safe procedure, used for diagnosis and treatment of intrauterine pathologies with a low percentage of complications. Some complications may be life threatening.

Case presentation: An otherwise healthy 44-year-old woman presented for hysteroscopic myomectomy under spinal anesthesia. A roller pump and a morcellator - type hysteroresectoscope were used along with normal saline as the distending solution with a perfusion pressure of $100 \mathrm{mmHg}$. Within 40 minutes, she complained of chest discomfort and presented tachypnea, tachycardia, hypertension and low oxygen saturation unresponsive to bag and mask ventilation with $100 \%$ oxygen. Massive facial edema developed rapidly. Orotracheal intubation was performed and pink frothy fluid came out of the ET. Fluid overload was suspected as the most likely diagnosis and the hysteroscopy was discontinued. Laboratory findings revealed severe respiratory acidosis and hypoxemia. She was treated aggressively with positive pressure ventilation, IV bicarbonate, diuretic and inotropic perfusions. A thorax x-ray confirmed the presence of massive pulmonary edema. After a highly negative fluid balance, the respiratory parameters improved very rapidly and she was extubated in the ICU 7 hours later.

Discussion: Fluid overload due to absorption of irrigation fluid is seen in approximately $0,2 \%$ of patients. Hypervolemic syndrome and hyperchloremic metabolic acidosis have been reported in NS overload. In this case, a positive fluid balance of almost 6.51 of NS was calculated; this was not identified before due to an error in the assembly of the circuit and bypass of the alarms thus leading to non-cardiogenic pulmonary edema, anasarca, respiratory acidosis, hypocalcaemia, coagulation and hemoglobin dilution, which resolved with forced diuresis and cardiopulmonary support. She was discharged after 7 days of the procedure.
\end{abstract}

Conclusion: General anesthesia and sedation can mask symptoms of irrigation fluid absorption. Anesthesiologists must monitor fluid balance and perfusion pressure, of the irrigation fluid as well as keep an open communication with the surgeon at all times to avoid this type of events. The use of normal saline is not without life threatening complications.

Keywords: Hysteroscopy, Pulmonary edema, Irrigation fluid
Volume I Issue 2 - 2014

\author{
Juan Carlos Sosa Nicora, Sergi Suarez, Diana \\ Lopez Cubillos, Josep Maria Bausili \\ Anesthesia and Critical Care Service, Hospital Igualada, \\ Consorci Sanitari de L'Anoia, Barcelona
}

Correspondence: Juan Carlos Sosa Nicora,Anesthesia Service and Critical Care Medicine, Hospital Igualada-Consorci Sanitari de L $\square$ Anoia, Av. Catalonia II, 08700, Igualada, Barcelona, Tel 938-075-500, Email koko97@gmail.com

Received: May 02, 2014 | Published: June 19, 2014
Abbreviations: ET, Endotracheal; ICU, Intensive Care Unit; NS, Normal Saline, IV, Intravenous

\section{Introduction}

Gynecological hysteroscopy is a relatively safe procedure used for diagnosis and treatment of uterine pathology with a low percentage of complications although some of them may be life threatening. These series of more severe complications include uterine perforation, laceration, and hemorrhage.

\section{Case presentation}

An otherwise healthy 44 year old woman $(159 \mathrm{~cm}, 54 \mathrm{~kg})$ was scheduled through day surgery regimen for a hysteroscopic myomectomy due to dysfunctional uterine bleeding. She was seen through the pre-anesthesia visit and her blood analysis, EKG and chest X-ray were unremarkable. She was assigned an ASA 1 status. The night before surgery, she received per OS $5 \mathrm{mg}$ of diazepam and $20 \mathrm{mg}$ of omeprazole. She presented to the Day Surgery ward calm and assured. When she entered the pre anesthesia room, a cefalic vein of the left hand was canalized with a number 20 abbocath; an IV drip with Plasmalyte ${ }^{\circledR}$ solution was initiated and administered $1 \mathrm{mg}$ of midazolam.

When she entered the operating room she was put in the sitting position and she received spinal anesthesia with a number 27 Whitacre needle from $\mathrm{BD}{ }^{\circledR}$ and was administered $60 \mathrm{mg}$ of prilocaine. She was then put in the dorsal decubitus position and cardiohemodinamic monitorization with NIBP, EKG and $\mathrm{SpO}_{2}$ was initiated. She preferred to be awake so no additional sedation was administered. A nasal cannula was applied according to protocol. A T10 level was confirmed before final positioning. Her baseline TA was 90/60 (mean arterial pressure of 70) and heart rate was 72 per minute. She kept spontaneous respiration and her Oxygen Saturation was $97 \%$ with supplementary $\mathrm{O}_{2}$ administered by nasal cannula at $3 \mathrm{lit} / \mathrm{min}$.

For the procedure, she was placed on gynecological position with a 10 degree trendelenburg tilt . The surgeon performed the procedure using a hysteroscopic morcellator and roller pump (Smith $\&$ Nephew $\left.{ }^{\circledR}\right)$, which provided a pressure-controlled infusion of 0 , $9 \%$ Normal Saline (NS) thus maintaining intrauterine pressures of 80 
$\mathrm{mmHg}$. For electrocautery a bipolar diathermy machine was used. Ten minutes after spinal anesthesia, a technical difficulty was encountered on behalf of the surgeon: he had poor vision on the monitor and with means of improving their vision they increased the perfusion pressure to nearly $100 \mathrm{mmHg}$ and bypassed the alarms of the morcellator machine. Fluid balance could not be kept due to rearrangement of the fluid administration circuit.

Forty minutes after spinal tap, the patient complained of chest tightness, and shortness of breath. The monitor revealed progressive tachycardia, (HR 122 bpm) moderate hypertension, (140/90 vs 90/60 baseline) at forty-five minutes she showed facial oedema and a drop in $\mathrm{O}_{2}$ saturation unresponsive to bag and mask ventilation with $100 \%$ oxygen. An allergic reaction was suspected and she was treated with $50 \mathrm{mcg}$ of IV adrenalin and $200 \mathrm{mg}$ of IV hydrocortisone with no improvement.

At fifty minutes we intubated the patient and pink frothy fluid returned through the endotracheal tube (ETT). Pulmonary oedema due to fluid overload was the most likely diagnosis and at that moment the operation was stopped. Treatment was initiated with positive pressure ventilation and PEEP was set to $+7 \mathrm{~cm} \mathrm{H} 2 \mathrm{O}$. An arterial line was placed in the right radial artery and the left subclavian vein was canalized with a two-lumen central vein catheter. Her vital signs were unstable and she presented an invasive arterial pressure of 110/90 with a heart rate of 122 . Her central venous pressure was $28 \mathrm{~cm} \mathrm{H}_{2} \mathrm{O}$. Her $\mathrm{SpO}_{2}$ was $76 \%$ and her extremities were cold and clammy. Blood gas results yielded severe respiratory acidosis and hypoxemia $(\mathrm{pH}$, 6.878; $\mathrm{HCO}_{3}$ 12.8, mmHg; $\mathrm{BE},-20,5 \mathrm{mM}$; pCO2, $72.9 \mathrm{mmHg}$; pO2, $25.8 \mathrm{mmHg}$ ). Plasma osmolality was $314 \mathrm{mosm} / \mathrm{kg}$. Nasopharyngeal body temperature measured $35^{\circ} \mathrm{C}$. Additional treatment included a furosemide infusion $(0,1 \mathrm{mg} / \mathrm{kg} / \mathrm{hr})$, bicarbonate infusion at $5 \mathrm{meq} /$ $\mathrm{kg}$ and continued for 8 hours along with convection temperature control. Fluid balance was recalculated with use of 27 liters of NS with a return of 20,5 liters in 30 minutes time. After the procedure was stopped, a total of 6, 5 liters of NS were absorbed in addition to 300 cc of IV Plasmalyte ${ }^{\circledR}$.

Approximately $500 \mathrm{cc}$ of fluid was recovered through the ETT. Chest X-ray confirmed the diagnosis (Figure 1). Then she was moved to the ICU ward and sedation with Propofol $3 \mathrm{mg} / \mathrm{kg} / \mathrm{hr}$ and Remifentanil 0, $5 \mathrm{mcg} / \mathrm{kg} / \mathrm{min}$ were initiated. During treatment with PPV and PEEP, arterial pressured dropped to $80 / 50$ and a dopamine infusion was initiated to keep MAP over $80 \mathrm{mmHg}$. 4 hours later, a second $\mathrm{ABG}$ showed improvement in the oxygenation and $\mathrm{pH}$ levels. Plasma electrolytes were: Na 144 mM, K 3, 0 mM, Cl 110 $\mathrm{mM} \mathrm{Ca}++5.6 \mathrm{mg} / \mathrm{dl}$. After a negative balance of 7 liters (Figures 2 and 3), respiratory parameters improved and she was extubated in the ICU 7 hours after the event. During the stay in the ICU she also presented dilutional anemia and coagulopathy that did not require transfusions (Hb 7, 4, Hct 26\%, INR1.6; PT 44.7\%, a PTT, 35 s.) She was discharged from the hospital 7 days later without any serious or irreversible neurological damage of any type.

Surgical hysteroscopies are performed in a fluid medium in order for the electrocautery instruments to work and to achieve optimal visualization. The type of solution used is determined by the surgeon and instrument utilized. Bipolar operative hysteroscopy can be performed using saline or Ringer's lactate solution. ${ }^{1}$ Surgical teams must be vigilant to the amount of fluid administered. Sometimes these difficulties are encountered due to poor team communication or lack of expertise amongst individuals that make up the team. ${ }^{2}$ In a series of 13600 of patients that underwent a hysteroscopic procedure, 5 cases
$(0,2 \%)$ of fluid overload were reported. ${ }^{3}$ Only a few cases have been published as case reports in the literature. Severity and diversity of the cases depends on the type of distension fluid. The use of NS has been linked to hypervolemic syndrome; hyperchloremic metabolic acidosis and non cardiogenic pulmonary edema. ${ }^{4}$ Cases have been reported of pulmonary embolism with the use of Ringer Lactate as distension fluid.

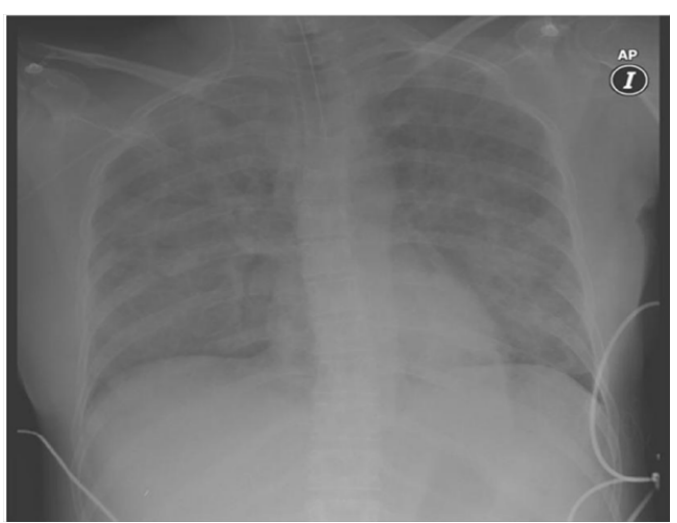

Figure I Chest X-ray diagnosis for confirmation of fluid content.

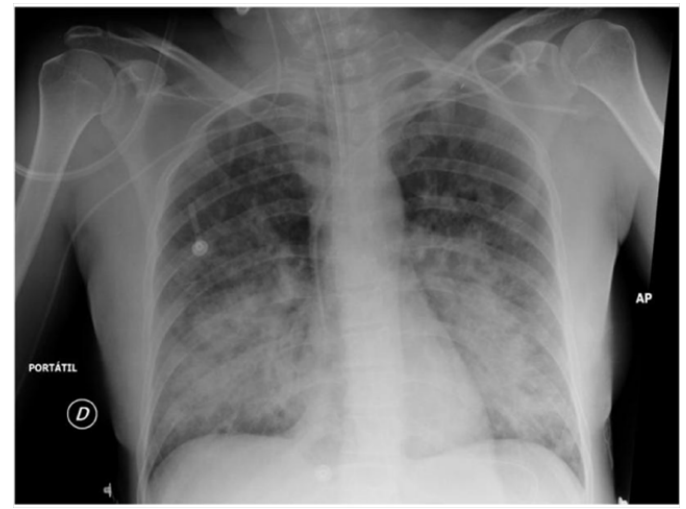

Figure 2 Chest $\mathrm{X}$-ray after respiratory parameters improved.

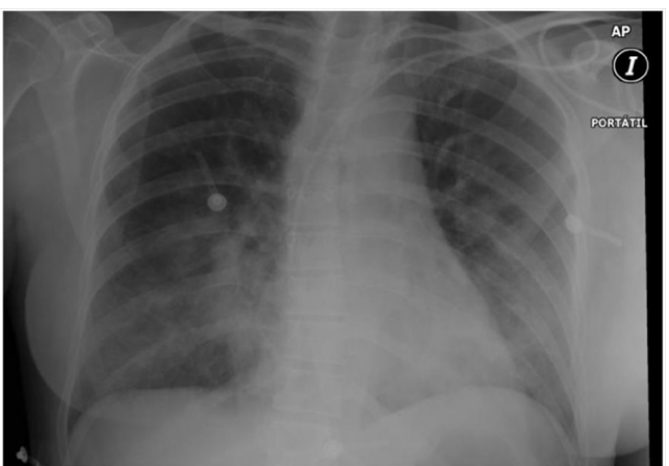

Figure 3 Chest $\mathrm{X}$-ray after a negative balance of 7 liters.

Morcelation technique comprises endometrial section through acicular blade while infusing the irrigation liquid and at the same time suctions the fluid and debry, thus maintaining a relatively stable intrauterine pressure. This type of technique leaves endometrial vessels open to the uterine cavity. When irrigation pressure exceeds the pressure in the vessels, fluid is absorbed. The amount of absorbed fluid is proportional to: ${ }^{6}$ 
i. Hydrostatic pressure of the irrigation fluid

ii. Number of exposed vessels

iii. Time of exposure

iv. Pressure gradient in the blood-irrigation fluid interface

We present the case of an iatrogenic absorption of 6, 51 of irrigation fluid, which went by unnoticed due to lack of communication with the surgeon, bypass of security alarms and in adequate fluid balance that resulted in non-cardiogenic pulmonary oedema, anasarca, severe neurological signs, severe respiratory acidosis that required aggressive reanimation measures. Fortunately, treatment led to a quick recovery of the life threatening state and discharge from the hospital 7 days later without permanent neurological damage.

\section{Conclusion}

The absorption of irrigation fluid during a hysteroscopic procedure must be considered as a possibility if a patient complains from chest discomfort and is accompanied by hemodynamic and respiratory instability, when undergoing regional anesthesia. Sedation and general anesthesia may mask the symptoms of absorption of irrigation media. This case presented mainly due to various medical errors. The anesthesiologist must monitor irrigation pressure, fluid balance and maintain communication with the surgeon at all times to avoid this type of complications. The use of normal saline as a distension media is not free of complications.

\section{Acknowledgements}

The authors would like to thank the people who work in the ICU at the Hospital de Igualada for their rapid response and their team work spirit.

\section{Conflicts of Interset}

None.

\section{References}

1. Jackson S, Lampe G. Operative hysteroscopy intravascular absorption syndrome. West J Med. 1995;162(1):53-54.

2. Bradley LD. Complications in hysteroscopy: Prevention, treatment and legal risk. Curr Opin Obstet Gynecol. 2000;14(4):409-415.

3. Jansen FW, Vredevoogd CB, van Ulzen K, et al. Complications of hysteroscopy: a prospective, multicenter study. Obstet Gynecol. 2010;96(2):266-270.

4. Schafer M, Von Ungern-Sternberg BS, Wight E, et al. Isotonic fluid absortion turing hysteroscopy resulting in severe hyperchloremic acidosis. Anesthesiology. 2015;103(1): 203-204.

5. Grove JJ, Shinaman RC, Drover DRet al. Noncardiogenic pulmonary edema and venous air embolism as complications of operative histeroscopy. J Clin Anesth. 16(1):48-50.

6. Pratesi R, Tonelli P, Torelli P, et al. Excessive reabsorption of irrigation fluid during operative hysteroscopy for uterine myoma. Minerva Anestesiol.1994;60(5):273-276. 